\title{
Neurogenic lower urinary tract dysfunction; management recommendations of the Neurologic Incontinence committee of the fifth International Consultation on Incontinence 2013
}

M.J. Drake*, A. Apostolidis, A. Cocci, A. Emmanuel, J. Gajewski, S.C.W. Harrison, J. Heesakkers, G. Lemack, H. Madersbacher, J.N. Panicker, P. Radziszewski, R. Sakakibara, J.-J. Wyndaele

\begin{abstract}
BACKGROUND:

Evidence based guidelines for the management of neurological disease and lower urinary tract dysfunction have been produced by the International Consultations on Incontinence (ICI). These are comprehensive guidelines, and were developed to have world-wide relevance.
\end{abstract}

AIMS:

To update the recommendations of the 4th ICI, 2009.

MATERIALS AND METHODS:

A series of evidence updates were performed by members of the working group in order to review the 2009 recommendations. The resulting guidelines were presented at the 2012 meeting of the European Association of Urology for consultation, and consequently amended to deliver evidence based conclusions and recommendations in 2013.

RESULTS:

The current review is a synthesis of the conclusions and recommendations, including the algorithms for initial and specialised management of neurogenic lower urinary tract dysfunction. The pathophysiology is categorised according to the nature of onset of neurological disease and the part(s) of the nervous system affected. Assessment requires clinical evaluation, general investigations and specialised testing. Treatment primarily focuses on ensuring safety of the patient and optimising quality of life. Symptom management covers conservative and interventional measures to aid urine storage and bladder emptying, along with containment of incontinence. A multidisciplinary approach to management is essential.

DISCUSSION:

The review offers a pragmatic review of management in the context of complex pathophysiology, and varied evidence base.

\section{Introduction}

Neurogenic lower urinary tract dysfunction (NLUTD) is a general term referring to the diverse effects of neurological disease on urinary tract function. The neurological regulation of the individual organs and their voluntary and reflex control is complex and mediated at several levels of the neuraxis. Thus, the potential for lower urinary tract (LUT) dysfunction in someone with neurological disease is substantial, and this may be one of the worst aspects of their condition for the affected individual, in terms of quality of life impact. This review sets out the conclusions of the Neurologic Incontinence panel of the fifth International Consultation on Incontinence regarding assessment and treatment of NLUTD, based on a comprehensive review of the published evidence base.

\section{Methods}

Using the previous review of the Neurologic Incontinence committee from the fourth International Consultation on Incontinence [1,2] as a baseline, an updated literature search of all published research was conducted from January 2008 to August 2012. The findings were presented at the annual scientific meeting of the European Association of Urology in 2012 for consultation, and modifications applied for the fifth edition of the ICI scientific report in 2013 [3]. Levels of evidence (LOE) and grades of recommendation (GOR) were derived according to 
the modified Oxford system developed by the International Consultations on Urological Diseases [4].

\section{Pathophysiology}

With a neurologic lesion, the type of NLUTD that arises depends on the site, extent and the evolution of the lesion. Key categories include;

1. Time of neurological dysfunction; congenital or perinatal, are considered distinct from those acquired later on, since the former never develop normal LUT function.

2. Likelihood of neurological progression; progressive conditions, contrasting with neurological lesions which are typically stable.

3. Extent of loss of neurological function.

4. Which part(s) of the nervous system is affected [5] (Table 1);

a) "Suprapontine", i.e. patients with lesions above the pons (forebrain or midbrain); they usually continue to have reflex contractions of the detrusor, but impaired cerebral regulation and central inhibition. This may lead to:

- Inappropriate timing of voids

- Inability to initiate voiding

- Neurogenic detrusor overactivity (NDO) and NDO-associated incontinence

- Voiding (where present) is usually synergic, but voluntary sphincter contraction to resist DO may give rise to "pseudo-dyssynergia"

b) Pontine. Brainstem lesions are rarely compatible with more than short-term survival. However, lesions could cause NDO or detrusor underactivity and even detrusor-sphincterdyssynergia (DSD) according to the location and extent of the lesion.

c) Suprasacral spinal cord.

- NDO and NDO-associated incontinence are common

- Detrusor- urethral sphincter dyssynergia (DSD) can also occur, often resulting in a significant post void residual (PVR) and "high pressure" bladder

- Poorly sustained detrusor contractions are often seen and lead to incomplete bladder emptying.

- Altered function of the sympathetic spinal centre in the thoraco-lumbar spinal cord may alter blood pressure control. Lesions above T6 spinal cord level may place the patient at risk of autonomic dysreflexia.

- Altered male ejaculatory function

d) Sacral spinal cord.

- Detrusor areflexia (loss of parasympathetic function)

- Stress urinary incontinence (SUI) due to sphincter deficiency (loss of Onuf's nuclei)

- Bladder compliance during bladder filling may be impaired.

e) Subsacral (cauda equina and peripheral nerves). There may be detrusor areflexia and/ or

SUI. Bladder compliance can be impaired. In diabetic neuropathy, detrusor overactivity can be seen in combination with the above.

The peripheral nerves and the lower spinal centres are often grouped under the term "lower motor neurones", as severe damage to these structures causes loss of contractile function. However, it is recognised that incomplete damage to peripheral nerves and the lower spinal centres can result in altered reflex activity and symptoms of bladder overactivity [6]. Elsewhere, the neurological lesions are termed "upper motor neuron lesions", where the consequences are impaired co-ordination and reflex function. This is a considerable simplification, and anatomically inexact, so the committee considers categorisation into lower versus upper motor neuron lesions should no longer be supported. 
- Neural lesions are described according to time of onset, risk of neurological progression, completeness, and neurological level.

- Complete suprasacral spinal cord lesions should describe whether enough sympathetic nucleus function is retained to place the patient at risk of autonomic dysreflexia.

\section{Initial assessment}

The aim of initial assessment is to ascertain;

1. The nature of the NLUTD, focussing on risk factors for major complications and associated symptoms

2. Previous management, availability of support, home circumstances, lifestyle factors, quality of life and desire for treatment

3. What limitations the neurological disease places on management options, e.g. cognition, mobility, toilet transfer, hand function and core support

4. Whether any other factors may contribute to LUT dysfunction, e.g. prostate enlargement or post-obstetric urethral hypermobility

5. Urinary tract infections (UTIs)/ bacteriuria, noting that symptoms may be unclear (especially where neurological disease affects sensory function), and that over-diagnosis is common

6. Medical, medication and surgical history, including whether any other co-morbidity may limit therapy options

7. Whether the neurological disease affects blood pressure control, leading to autonomic dysreflexia or postural hypotension

8. Bowel and sexual function

9. Risk of decubitus ulcers

An overview of initial assessment as part of initial management is given in Figure 1.

\section{Specialised assessment}

\section{Urodynamic tests}

Urodynamic techniques evaluate multiple functional parameters in NLUTD, aiming to define bladder and outlet function during bladder filling and emptying. The specific information needed depends on the circumstances of each individual patient, and needs to be defined before initiating testing. Safety factors have to be addressed prior to the test, and consideration of infection prophylaxis and prevention of autonomic dysreflexia in relevant patients is important in NLUTD. Relevant investigations include; voiding diary, urodynamic studies (cystometry, electromyography (EMG), uroflowmetry, pressure-flow study), diagnostic imaging with voiding cystourethrography and ultrasonography. The use of multichannel cystometry and pressure flow studies with synchronous imaging (videourodynamics) offers suitable testing in most patients. The International Urodynamic Basic Spinal Cord Injury (SCI) dataset [7] recommends data to be included in the urodynamic evaluation of patients with SCI. Variables included comprise;

- Bladder sensation during filling cystometry

- Detrusor function and compliance during filling cystometry

- Sphincter function during bladder filling

- Detrusor/ sphincter function during voiding

- Detrusor leak point pressure in patients with impaired bladder compliance

- Cystometric bladder capacity and post-void residual

These are also likely to be relevant in other forms of NLUTD. Additional urodynamic testing modalities may be considered in selected circumstances. 
Videourodynamics (VUDS) offers visualisation of bladder and outlet, including pelvic floor support, to provide anatomical and functional information during filling and voiding. Such testing is informative regarding risk factors for upper urinary tract problems, mechanism(s) of incontinence, and mechanism(s) of voiding dysfunction. Without such information, caution is needed in interpreting cystometry and pressure flow study results. The presence of vesicoureteric reflux and of reflux of X-ray contrast into the prostatic ducts may also be detected during a video-urodynamic assessment.

Filling rate for cystometry should be initiated at a slow rate (e.g. $10 \mathrm{ml} / \mathrm{min}$ ); medium fill rates can be used in patients who are known to have a normal or high functional bladder capacity.

- Findings of urodynamic tests can be difficult to anticipate from clinical assessment alone in NLUTD (LOE 2).

- A combination with EMG and /or imaging adds to the diagnostic possibilities (LOE 2).

- Filling rate can influence the outcome of several urodynamic parameters (LOE 2).

- Pressure development in the bladder is one of the important parameters to be studied and a high detrusor leak point pressure is a risk factor for renal deterioration (LOE 2).

- Sensation of filling may be preserved despite spinal abnormality (LOE 2).

Recommendations

- Urodynamic tests should selectively be employed to supplement clinical assessment in determining management in NLUTD.

- Methods of Urodynamic testing in NLUTD should follow International Continence Society recommendations $[8,9]$.

- EMG of the urethral sphincter can be considered as a diagnostic method in patients with NLUTD and neurologic urinary incontinence (GOR B).

- There are some arguments that nerve conduction studies can be useful in the further differentiation of the nerve deficits in cases of a suspected neurological cause for bladder dysfunction (GOR C).

- Somatosensory evoked potentials can be of use in the further diagnosis of neurological deficits related to LUT dysfunction (GOR C).

- Sympathetic skin responses seem promising and the further study of them are recommended for the evaluation of the LUT sympathetic innervation (GOR B).

An overview of specialised assessment and therapy is given in Figure 2.

\section{Conservative treatment}

A range of measures aimed at symptom management or containment can be considered. Some are experimental, and need additional development before they can be recommended for routine practice. Others are no longer recommended, but are included in the current report because patients may have been established on the method previously, and do not wish to convert to an alternative method. Individuals with NLUTD due to SCI often change their method of bladder management during the protracted follow up necessary, influenced by a range of factors including renal function [10] (LOE 2).

\section{Behavioral therapy}

- Triggered reflex bladder emptying

- Bladder expression (Credé) techniques are no longer recommended due to the high rate of renal complications that are seen with this type of bladder emptying.

- Bladder emptying by abdominal straining (Valsalva).

- Toileting assistance

\section{Catheters and appliances}

- Clean intermittent catheterization (CIC) 
- Indwelling catheterization. Suprapubic catheterisation avoids the serious complication of urethral trauma which is a particular risk in patients with impaired LUT sensation or have behavioural difficulties.

- External penile appliances (penile sheath systems) and incontinence pads.

\section{Pharmacotherapy}

\section{Electrostimulation}

- Electrical Neuromodulation

- Repetitive transcranial magnetic stimulation

- Deep brain stimulation

- Electrical stimulation of the pelvic floor musculature

- Intravesical electrical stimulation

\section{Safety considerations}

Some specific situations require particular attention in NLUTD;

1. Complications of urodynamic testing are rare, but antibiotic prophylaxis can be advocated (LOE 2).

2. Autonomic dysreflexia; acute life-threatening risk can result from severe hypertension in patients with high spinal cord dysfunction (typically SCI above T6). Various triggers of episodes of autonomic dysreflexia are recognized, including iatrogenic urological procedures. Routine blood pressure monitoring during investigations or invasive therapy is therefore appropriate.

3. Allergy to latex needs appropriate preventive and therapeutic arrangements to be in place in case of anaphylactic reaction.

4. Urodynamic features should be used to gauge potential impact on a patient's renal function as a consequence of NLUTD.

- Bladder compliance; impaired compliance with a high detrusor leak point pressure is a high risk situation needing early intervention

- DSD

- Vesico-ureteric reflux

Exact parameters for acceptable values of urodynamic parameters have not been established.

- Reflex bladder emptying is based on a non-physiological sacral reflex. It is potentially dangerous and has a limited role in managing the NLUTD (LOE3).

- Bladder emptying using external expression, is hazardous for the urinary tract if there is functional obstruction at the level of the pelvic floor (LOE 3).

- Bladder emptying by abdominal straining is contraindicated if it creates a high intravesical pressure, particularly in association with reflux into the ureters or seminal vesicles.

Hernias, pelvic organ prolapse, haemorrhoids and urethral pathology (strictures) are relative contraindications (LOE 3).

- Bladder expression and abdominal straining may adversely affect a flaccid pelvic floor, potentially exacerbating incontinence (LOE 3).

\section{Recommendation}

- Any unit managing patients with SCI should be appropriately set up to manage acute autonomic dysreflexia.

\section{Symptom management}

The detrusor and sphincter are often both affected by the neurogenic lesions. In many patients a storage problem, leading to incontinence, is associated with an emptying problem; therefore both aspects have to be considered. Therapy of neurogenic incontinence is primarily a conservative one. Timed bladder emptying, controlled fluid-intake and avoidance of urinary tract infections are needed for successful treatment. 
In supraspinal lesions, NDO is mostly associated with normal sphincter function; NDOassociated incontinence is the main problem for many patients and antimuscarinic therapy together with behavioural treatment is the method of choice, more so in patients with cognitive impairment.

Spinal lesions mostly cause simultaneous dysfunction of the detrusor and the sphincter. In suprasacral lesions, DO often occurs with an overactive/ dyssynergic sphincter. For these patients, spontaneous reflex voiding may be possible, but often emptying is incomplete. The mainstay of treatment in current practice is intermittent catheterization (IC), undertaken by the patient or carer. However, to achieve low pressure LUT urine storage and continence between catheterisations, additional pharmacotherapy may be necessary. If incontinence persists, and if operative procedures are not indicated or possible, containment products will be needed. The indwelling catheter remains an option for conservative therapy, and can offer acceptable quality of life outcomes and long-term protection of the upper urinary tract. Most experts regard indwelling catheterisation as being associated with significant problems [10]. While suprapubic catheters are generally preferred over urethral, there is little published evidence on which to base practice, but it is very clear that urethral catheters can be associated with severe urethral trauma. Urethral leakage can persist despite continuous drainage with a SPC, so surgical treatment of the stress incontinence, or even bladder neck closure, may need to be considered.

For complete conus lesions, areflexia of the detrusor with areflexia of the sphincter is characteristic. Sphincter incompetence causes neurogenic SUI and may be combined with dribbling incontinence if adequate emptying is not achieved. Penile sheath systems or incontinence pads are often necessary, and consideration of suitability of surgery may be needed.

Sometimes, areflexia of the detrusor may be combined with overactivity of the sphincter, in which case CIC may be appropriate, or an indwelling (suprapubic) catheter may be needed. DO with sphincter areflexia can occur in myelomeningoceles; bladder relaxant agents may be trialled, but conservative treatment alone is generally unable to restore continence, so appliances or operative treatment must be considered.

Cauda equina and peripheral nerve lesions are often incomplete. Acontractile detrusor may be combined with a normally functioning external striated sphincter, or the reverse combination, may be present.

Conclusions

- Costs of appliances and of adjuvant therapies (pharmacotherapy, surgery, urethral prosthesis etc.) have to be evaluated (LOE 2).

- Treatment of co-existing sphincteric spasticity/ bladder neck obstruction and comorbidity should be taken into consideration (LOE 1 and 2)

- Alpha-blockers, sphincterotomy or botulinum neurotoxin-A (BoNT-A) may reduce the outflow resistance, but may also induce or increase urinary stress incontinence (LOE 3).

- Behavioural techniques should be used in conjunction with other therapies (pharmacological treatment, catheterisation) (LOE 2)

- When appropriate, toileting assistance should be used to improve continence of neurologic impaired patients (LOE 3)

- Prompted voiding may be able to decrease incontinence episodes. Patients likely to benefit from the technique are those with less cognitive impairment and higher dependency (LOE $2 / 3$ ).

Recommendations

- Triggered bladder emptying could be recommended only for patients whose situation has proven to be urodynamically safe and stable, and who can manage reflex incontinence. 
- Triggered bladder emptying can be considered for patients after sphincterotomy and/or bladder neck incision and/or alpha-blockers and/or intrasphincteric botulinum toxin injections, in order to improve spontaneous reflex voiding (GOR C).

- Reflex bladder emptying can be recommended only if an adequate follow-up is guaranteed (GOR C)

- Before recommending bladder emptying by abdominal straining it must be proven that the LUT is urodynamically safe. (GOR B)

- Exclude contraindications, such as vesico-ureteric reflux, pelvic organ prolapse, hernias, urethral pathology and symptomatic UTIs before recommending this type of bladder emptying. (GOR B)

- In general, CIC should be considered to be the most appropriate means of achieving complete bladder emptying in most patients with neurogenic bladder-sphincter dysfunction and impaired bladder emptying. (GOR B)

- Adjunctive therapy of outflow obstruction can be considered. (GOR B).

- Behavioural techniques are a suitable component of the rehabilitation program for each individual. (GOR C)

- There are no guidelines or consensus on suitable intervals for bladder emptying. They should ideally be derived from the voiding diary and other related factors (bladder volume, fluid intake, post-void residual urine volume, urodynamic parameters). (GOR C)

- The mental status of a patient must be taken into consideration, and a rehabilitation program realistically tailored to the patient's possibilities. (GOR B/C).

\section{Catheters and appliances}

Bladder drainage with a catheter is necessary in a substantial proportion of people with NLUTD. Complications can include;

- Encrustation may be predictive of bladder stones. Cystoscopy is the only reliable way to detect bladder stones; abdominal $\mathrm{x}$-ray is not a reliable way to diagnose the presence of bladder stones (LOE 3).

- Urethral trauma (traumatic hypospadias in men, patulous urethra in women) from the use of indwelling urethral catheter in patients with NLUTD.

- Urinary tract infection. Urethral flora may be a bacterial source for the development of urinary infection (LOE 3), but low bacterial concentrations in the urine $(<105 \mathrm{cfu} / \mathrm{L}$ ) of patients on IC might be due to contamination (LOE 3). Cranberry extract, methenamine hippurate or phosphorus supplements were not found to be effective in acidifying urine or preventing urinary tract infection (LOE 2). A weekly oral cyclic antibiotic seemed efficacious in preventing UTI (LOE 3). Bladder irrigation was not effective in reducing bacteriuria in persons using indwelling catheterization (LOE 2). In people with spinal cord dysfunction, combined IC during the day time with indwelling catheter at night time showed less urinary infection than IC with incontinence or indwelling catheterization (LOE 3).

Conclusions for IC

- IC in the neurogenic bladder is effective and safe for short- and long- term use. (LOE 1)

- Complications such as UTI are regularly seen and seem to be related to both the catheterization itself and the pre-existing LUT condition (LOE 2)

- Urethral and bladder complications seem to increase in the long- term (LOE 3)

- In order to reduce and prevent complications, appropriate materials and correct techniques should be taught and performed (LOE 3)

- Adequate frequency of IC, a non-traumatizing technique and suitable materials are the key factors for a successful outcome (LOE 2).

Conclusions for indwelling catheters 
- Long-term indwelling urethral catheter use in neurologic patients can predispose to complications (LOE 2).

- The catheter chosen should not be so large or rigid as to traumatise the urethra, and should have the maximum luminal diameter possible (LOE 4).

- All silicone catheters may be a suitable choice, in view of the comparatively large luminal size for some models (LOE 4).

- $\quad 5-10 \mathrm{ml}$ self-retaining balloons should be used to minimise the pressure effect on the bladder neck (LOE 4).

- Closed drainage systems are associated with lower infection risk (LOE 1).

- Frequency of change largely depends on time to blockage, which is influenced by catheter materials and lumen, patient factors and infection (LOE 3).

- Suprapubic catheter is superior to urethral catheter if long-term catheterisation is needed, but IC is the first line intervention (LOE 3).

- Suprapubic catheter is a safe and effective short-term management of urinary retention (LOE 3).

Conclusions for penile sheath appliances

- Penile sheath appliances facilitate urinary containment of incontinence in some male patients with NLUTD (LOE 3)

- Complications may be less if technique, hygiene, replacement and maintenance of low bladder pressures are optimised (LOE 3).

Recommendations for IC

- IC is the first choice treatment for those with inability to empty the bladder adequately and safely in neurogenic voiding dysfunction. It is a valuable tool for achieving continence (GOR A)

- Proper education and teaching are necessary to achieve a good outcome (GOR B).

- To prevent and reduce complications, a non-traumatizing technique (external lubricant or lubricant coated catheters) with adequate frequency of catheterization and complete emptying should be achieved (GOR B).

- Annual follow-up is needed (GOR B/C).

- It is not currently possible to state whether any IC method is advantageous (GOR D) and further research on the topic is strongly recommended.

Recommendations for indwelling catheters

- Silicone or hydrogel-coated catheters are preferable (GOR A/B).

- Use sterile materials and aseptic technique, and routine catheter care in the context of a closed drainage system (GOR C/D).

- Catheters should be changed regularly, to try to pre-empt obstruction or infection (GOR C/D).

- Bladder irrigation and antibiotic prophylaxis are not recommended as a routine infectioncontrol measure. Symptomatic UTI should be treated with narrowest spectrum antibiotic possible, according to local microbiology practice (GOR B).

- Patient education on daily cleanliness and hygiene care are mandatory (GOR C).

- Short-term indwelling catheter during the acute phase of neurological injury is a safe management for neurologic patients (GOR B).

- Long-term indwelling catheter may be safe only if a careful check-up of urodynamic, renal function, and upper and lower tract imaging are performed (GOR B).

- Cystoscopy and biopsy is necessary for those with visible hematuria, or chronic symptomatic UTI refractory to therapy (GOR C).

- Consider the use of antimuscarinics in individuals with suprasacral lesions using chronic indwelling catheters (GOR C). 
- Patient comfort, convenience, sexuality and quality of life need to be considered (GOR C). Recommendations for penile sheath appliances

- Size selection should consider control of leakage, and prevention of penile compressive effects (GOR B).

- Regular bladder emptying with low bladder pressures and low post void residual should be confirmed (GOR B).

\section{Pharmacotherapy}

- Antimuscarinic drugs improve storage function (LOE 1).

- Antimuscarinic drugs have a high incidence of side effects (dry mouth, constipation, urinary retention, etc.). Antimuscarinics with longer duration of action and controlledrelease formulations have significantly less side effects compared to immediate-release oxybutynin (LOE 1).

- High doses of oxybutynin have been used to treat patients with neurogenic bladder dysfunction (LOE 3).

- Intravesical instillation of oxybutynin may be an alternative route of administration (LOE 4).

- Onabotulinumtoxin-A injection into the detrusor muscle improves clinical and urodynamic parameters (LOE1), and has been approved as second-line treatment for urinary incontinence associated with NDO in patients with inadequate response to or intolerance of an anticholinergic.

- Repeat intradetrusor injections of Onabotulinumtoxin-A provide sustained clinical benefits (LOE3)

- Treatment with intradetrusor Onabotulinumtoxin-A is considered overall safe, with increased post-void residual and need for post-treatment CIC being the most common adverse event (LOE1).

- BoNT-A can be considered for the treatment of DSD in spinal cord injury patients (LOE2). The effect of BoNT-A intrasphincteric injections are short-lived and therefore the longterm safety and efficacy of the treatment is uncertain. However, on the basis of one LOE1 study, BoNT-A does not provide significant benefit for the treatment of DSD in MS patients. Further evidence is needed before recommendation for its use is possible.

- There is no adequately designed controlled study of any drug for neurogenic sphincter deficiency.

\section{Recommendations}

- Antimuscarinic drugs should be recommended for the treatment of neurogenic detrusor overactivity (GOR A). Titration of the dosage of these drugs individually should be done for optimal balance of therapeutic and adverse effects. If one drug is not tolerated, another drug should be tried (GOR C/D).

- Onabotulinumtoxin-A should be offered as a treatment option for incontinence associated with NDO (GOR A).

- Further research is needed on long-term outcomes and safety, administration techniques, the bio-equivalence of the various preparations of BoNT-A, the concomitant use of anticholinergic drugs, mechanisms of action, and wider effects (GOR A)

- Vanilloid intravesical therapy still remains experimental and therefore is not recommended except within clinical trials (GOR C/D)

- For decreasing outlet resistance in neurogenic bladder alpha-adrenergic antagonists may be used (GOR B/C).

- BoNT-A may be considered for DSD in spinal cord injury patients (GOR B)

- For neurogenic sphincter deficiency, no effective drugs are available up to now; further research is needed (GOR D). 
- For detrusor areflexia no effective drugs are available up to now; further research is needed

\section{Electrostimulation}

- Electrical neuromodulation is not first line treatment for neurogenic DO. There are some limited reports showing that it may be beneficial (LOE 3).

- Automatic, event-driven electrical stimulation in the treatment of neurogenic DO is feasible (LOE 3).

- Chronic pudendal nerve stimulation is feasible. Neurophysiological guidance seems to be mandatory to place the lead near the pudendal nerve (LOE3).

- Percutaneous tibial nerve stimulation gives improved clinical and urodynamic parameters (LOE3), but its role is not established.

- Enhancing corticospinal tract excitability by repetitive transcranial magnetic stimulation might be useful to ameliorate detrusor contraction and/or urethral sphincter relaxation in MS patients with bladder dysfunction (LOE3).

- Deep brain stimulators may affect urodynamic parameters (LOE3)

- There is no study published which deals with electrical stimulation via anal or vaginal plugs could be able to improve the strength of pelvic floor musculature in neurological disease (LOE 4)

- Basic research during the last decade supports the underlying working concept of intravesical electrical stimulation (LOE 3). The results reported in the literature are controversial, mainly because of varied inclusion and exclusion criteria (LOE 3). In the only sham-controlled study the treatment period was short and the inclusion and exclusion criteria were not fully defined (LOE 3).

\section{Recommendations}

- If pharmacotherapy fails to improve NDO, electrical neuromodulation may be optional in patients with NDO (GOR C/D)

- Although the setup for conditional, event-driven electrical stimulation is not suitable in a clinical setting, the treatment modality is promising and it warrants further investigation (GOR D).

- Further studies on chronic pudendal nerve stimulation must be carried out to identify the best stimulation parameters and to verify the long term results (GOR D)

- The thalamus may be a promising target for the development of new therapies for LUT dysfunction. Further investigation on this matter is needed before its potential role can be elaborated.

- In patients with incomplete denervation and some voluntary contraction of the pelvic floor muscle and the striated sphincter, electrical stimulation may be an option to improve pelvic floor function, thus improve incontinence (GOR C/D)

- Intravesical electrotherapy may improve NLUTD, inducing bladder sensation and the urge to void (GOR B/C)

- Intravesical electrical stimulation is the only available option to induce/improve bladder sensation and to enhance the micturition reflex in patients with incomplete central or peripheral nerve damage, but corroborating controlled evidence is needed (GOR B)

- Selection of patients is crucial and intravesical electrical stimulation should be applied only if afferent fibres between the bladder and the cortex are still intact and if the detrusor muscle is still able to contract (GOR B). The ideal indication is the neurogenic hyposensitive and hypocontractile detrusor (GOR C) 


\section{Interventional procedures}

Surgery to treat neurogenic detrusor overactivity

- For severe neurogenic DO, bladder surgery using intestinal segments may be considered. In idiopathic DO/ OAB, sacral neuromodulation (SNM) is an alternative to intravesical Onabotulinumtoxin-A treatment, but the role of SNM in NLUTD is not established. Additional research is needed to ascertain the potential contribution of SNM for NLUTD in routine practice.

- Bladder denervation, for example peripheral nerve blockade and dorsal rhizotomy, is mainly reserved for those suffering complete spinal cord injuries, and has not achieved consistent results.

\section{Recommendations}

- Ileum, colon, stomach or ureter may be used for bladder augmentation, but the ileum seems to give the best results in terms of ease of use, risk of complications and efficacy (GOR B). Limited data is available concerning gastrocystoplasty and ureterocystoplasty in adults (GOR D)

- When the bladder suffers a significant compliance defect, supratrigonal cystectomy and reconstruction is preferable to clam cystoplasty (GOR B).

- Patients should be informed that the most frequent and serious complications are bladder calculi and perforation at the bladder/bowel junction, usually caused by overdistension of the bladder (GOR B).

- Bladder augmentation may have sequelae such as intestinal transit disorder, and patients should be informed of this before surgery (GOR C).

- The body of evidence concerning detrusor myomectomy in neurological patients is controversial. Therefore, detrusor myomectomy should not be recommended in these patients with impaired bladder function (GOR D)

- Bladder augmentation using biomaterials or tissue engineering is promising, but the preliminary results need to be confirmed by larger studies (GOR D)

- Due to risk of complications, regular follow up is needed GOR (B)

- Sacral neuromodulation can have an inhibitory effect on neurogenic DO (GOR C)

- Indications for sacral neuromodulation in the care of DSD in NLUTD are still not established (GOR D).

- No peripheral bladder denervation technique has passed the test of time (GOR D)

- Injections of neurolytic products to treat detrusor overactivity should be abandoned, since they are ineffective in the medium and long term and expose patients to morbidity (GOR A)

- Sacral dorsal rhizotomies need to be quite extensive to treat neurogenic DO successfully. So they may be performed only in patients with lower limb neurological impairment (GOR B)

- In certain situations, dorsal rhizotomies can be undertaken in association with ventral root stimulators (Brindley's technique) or even with continent cystostomy (GOR B)

- Electrostimulation of the anterior sacral roots is a valid option for managing NLUTD in patients with complete suprasacral SCI, with long-term follow-up (GOR B)

- It is usually combined with division of the dorsal S2,3,4 nerve roots (GOR B)

- Dorsal rhizotomy exposes men to loss of reflex erection and ejaculation; women are likely to lose reflex vaginal lubrication(GOR B) 
- It is vital to assess the patient carefully before implantation so as to determine whether he/she will be able to manipulate the device and manage their bladder emptying; the input of carers may be needed in some cases (GOR B).

\section{Surgery to treat poor bladder emptying}

\section{Outlet dyssynergia}

DSD is a characteristic feature of suprasacral and infrapontine lesions.

- The aim of sphincterotomy is to assist bladder emptying using reflex micturition or, in a few patients, abdominal straining, into a penile sheath appliance, thus protecting the upper urinary tract. Endoscopic sphincterotomy has been the technique of choice for patients who cannot or do not want to do IC. It is invasive, irreversible and the patient has no adaptation period (LOE3).

- Prosthetic sphincterotomy using a urethral endoprosthesis (or stent) is an alternative which can be associated with significant complications.

\section{Recommendations}

- Whatever type of sphincterotomy is chosen (surgical or prosthetic):

- Patients must think carefully about the different modes of micturition possible for them (GOR A).

- The few studies reporting long-term results of sphincterotomy demonstrate the vital importance of regular patient monitoring for the recurrence of DSD or blockage (GOR B).

- This mode of micturition is contraindicated in women, and in men with difficulty in maintaining a condom catheter (GOR B).

- Men who wish to have children should be warned of the risk of ejaculatory duct obstruction (GOR B).

- For patients who have chosen surgical sphincterotomy:

$\circ$ The reference technique involves an elective 11,12 or 1 o'clock incision of the urethral sphincter (GOR B).

- Although surgical sphincterotomy is the accepted reference treatment for neurogenic DSD, analysis of the literature highlights the lack of reliable efficacy and reproducibility criteria for the technique (GOR B).

- For patients who have chosen prosthetic sphincterotomy:

- Different types of stent are used, depending on whether sphincterotomy is temporary or permanent. Stents are complementary, and different designs can be used for different situations (GOR B).

- Careful follow-up is needed when leaving a permanent urethral stent (GOR B).

- Published data is inadequate to support a recommendation on the use of bladder neck incision in patients with inadequate bladder emptying due to detrusor-bladder neck dyssynergia (DBND), either as a first-line procedure or as a complementary approach when DBND is recognised following permanent urethral stenting. Further studies are needed.

\section{Surgery to increase detrusor strength}

Some teams have suggested placing rolled strips of muscle around the bladder. Some authors have also suggested a strip of rectus abdominis muscle. This is easier to perform and may be used essentially for reconstructive surgery, such as in bladder exstrophy (LOE 4).

\section{Recommendations:}

- Latissimus dorsi myoplasty on the bladder is a promising technique that needs to be validated further (GOR C). 


\section{Stress urinary incontinence due to sphincteric incompetence}

Patients with lesions of the conus medullaris, the cauda equina or peripheral sacral nerves are at risk of developing neurogenic SUI. In all forms of NLUTD, direct sphincter injury may result from urethral catheter trauma, or previous interventions such as sphincterotomy or dorsal rhizotomy. In women, SUI may be present for non-neurogenic reasons such as urethral hypermobility due to previous pregnancy and childbirth.

Recommendations:

- Patients with stress incontinence in association with NLUTD require careful assessment in order to plan appropriate management. Video-urodynamic study must be used to evaluate both bladder and sphincter function (GOR C).

- The clinical assessment must also evaluate the degree of patient handicap in order to determine whether they can perform self-catheterization, or whether an alternative means of emptying the bladder will be required (GOR D).

- Patients require careful preoperative counselling with respect to the benefits and risks of different operative approaches

- Autologous slings can be used to treat neurogenic stress incontinence (GOR B). The use of synthetic slings and tapes is not supported by an adequate evidence base at present (GOR C).

- Artificial urinary sphincter can be used to treat neurogenic stress incontinence (GOR A).

- Bladder neck reconstruction can be used to treat neurogenic stress incontinence (GOR D).

- Bulking agents can be used to treat neurogenic stress incontinence when there is a demand for a minimally invasive treatment (GOR D). The patient should be aware that the technique has a low success rate.

- Bladder neck closure should be offered to patients who have persistent neurogenic stress incontinence where alternative treatments have either failed or are likely to fail (GOR B)

\section{Urinary diversion}

\section{Continent cutaneous diversion}

- The use of a continent catheterisable abdominal conduit (CCAC) should be considered in the context of a multidisciplinary evaluation involving the urologist and a neurologist or rehabilitation doctor, as well as stomatherapy nurses or occupational therapists for estimating patient catheterization capabilities (GOR A)

- Use of the appendix to carry out CCAC is the standard method in children, but few long term data are available in adults (GOR C). The appendix may have a short mesentery, so a reconfigured ileal segment is often needed.

- If the patient has undergone an appendicectomy, the use of a segment of the small intestine can be proposed, with slightly poorer short term results (GOR C)

- Long term follow up after CCAC is needed to have a better idea of the long term results of the various procedures (GOR C).

\section{Non-continent cutaneous diversion}

- Non-continent urinary diversion is the last resort for patients with neurogenic bladder (GOR A).

- The risks and benefits of carrying out a simultaneous cystectomy should be discussed with the patient because of the risk of later complications from the defunctioned bladder (GOR B) 
- It may be indicated for urological dysfunction or in the event of a motor handicap that prevents other modes of LUT management (GOR C).

- Ileal conduit urinary diversion has the best long-term results for non-continent diversion, if the following pre- and peri-operative precautions are taken (GOR B):

- Pre-operative identification of optimal location for the stoma site, with wheelchair test, if necessary.

- Avoidance of the use of an unnecessarily long intestinal segment.

- Minimal dissection of the ureters.

- There are several reports of good results for ileovesicostomy, but the medium-term results need to be confirmed in the long term. Quality-of-life studies should also be performed (GOR C)

- Vesicostomy may be a useful temporary solution, particularly for children (GOR D)

- Cutaneous ureterostomy shouldn't be used for non-continent urinary diversion in adult patients because of the rate of long term complications (GOR B).

\section{Conclusions}

The pathophysiology of NLUTD is categorised according to the nature of onset of neurological disease likelihood of progression, and where the nervous system is affected. Assessment requires history and examination, general investigations and specialised testing. Treatment primarily focusses on ensuring safety of the patient. Symptom management covers conservative and interventional measures to aid urine storage and emptying, with containment and practical support an essential element in achieving acceptable quality of life and dignity. A multidisciplinary team approach to management is essential.

\section{Acknowledgements}

JNP acknowledges support from the University College London Hospitals NHS Foundation Trust (UCLH)/University College London (UCL) Department of Health National Institute for Health Research (NIHR) Biomedical Research Centres funding scheme. 


\section{References}

1. Abrams P, Andersson KE, Birder L, Brubaker L, Cardozo L, Chapple C, Cottenden A, Davila W, de Ridder D, Dmochowski R et al: Fourth International Consultation on Incontinence Recommendations of the International Scientific Committee: Evaluation and treatment of urinary incontinence, pelvic organ prolapse, and fecal incontinence. Neurourol Urodyn 2010, 29(1):213-240.

2. Abrams P, Wein A, Cardozo L, Khoury S: Incontinence. The fourth International Consultation on Incontinence. Paris: Health Publications Ltd; 2009.

3. Abrams P, Cardozo L, Khoury S, Wein A (eds.): Incontinence: the fifth International Consultation on Incontinence: Paris, France: European Association of Urology/ International Consultation on Urological Diseases; 2013.

4. Abrams P, Khoury S: International Consultation on Urological Diseases: Evidencebased medicine overview of the main steps for developing and grading guideline recommendations. Neurourology and Urodynamics 2010, 29(1):116-118.

5. Swain S, Hughes R, Perry M, Harrison S, Guideline Development G: Management of lower urinary tract dysfunction in neurological disease: summary of NICE guidance. $B M J$ 2012, 345:e5074.

6. Podnar S, Trsinar B, Vodusek DB: Bladder dysfunction in patients with cauda equina lesions. Neurourol Urodyn 2006, 25(1):23-31.

7. Biering-Sorensen F, Craggs M, Kennelly M, Schick E, Wyndaele JJ: International urodynamic basic spinal cord injury data set. Spinal Cord 2008, 46(7):513-516.

8. Schafer W, Abrams P, Liao L, Mattiasson A, Pesce F, Spangberg A, Sterling AM, Zinner NR, van Kerrebroeck P: Good urodynamic practices: uroflowmetry, filling cystometry, and pressure-flow studies. Neurourol Urodyn 2002, 21(3):261-274.

9. Abrams P, Cardozo L, Fall M, Griffiths D, Rosier P, Ulmsten U, van Kerrebroeck P, Victor A, Wein A: The standardisation of terminology of lower urinary tract function: report from the Standardisation Sub-committee of the International Continence Society. Neurourol Urodyn 2002, 21(2):167-178.

10. Drake MJ, Cortina-Borja M, Savic G, Charlifue SW, Gardner BP: Prospective evaluation of urological effects of aging in chronic spinal cord injury by method of bladder management. Neurourol Urodyn 2005, 24(2):111-116. 


\begin{tabular}{|l|l|l|l|}
\hline & $\begin{array}{l}\text { Congenital \& perinatal } \\
\text { lesions }\end{array}$ & $\begin{array}{l}\text { Acquired, stable } \\
\text { conditions }\end{array}$ & $\begin{array}{l}\text { Acquired, progressive } \\
\text { conditions }\end{array}$ \\
\hline $\begin{array}{l}\text { Brain and } \\
\text { brainstem }\end{array}$ & Cerebral palsy & Stroke, Head injury & $\begin{array}{l}\text { Multiple sclerosis, } \\
\text { Parkinson's disease, } \\
\text { Dementia, } \\
\text { Multiple System Atrophy* }\end{array}$ \\
\hline $\begin{array}{l}\text { Suprasacral } \\
\text { spinal cord }\end{array}$ & $\begin{array}{l}\text { Hereditary spastic paraparesis, } \\
\text { Spinal dysraphism* }\end{array}$ & Trauma & $\begin{array}{l}\text { Multiple sclerosis* } \\
\text { Spondylosis with } \\
\text { myelopathy }\end{array}$ \\
\hline $\begin{array}{l}\text { Sacral } \\
\text { spinal cord }\end{array}$ & $\begin{array}{l}\text { Spinal dysraphism, } \\
\text { Sacral agenesis, } \\
\text { Ano-rectal anomaly }\end{array}$ & Conus injury & \begin{tabular}{l} 
Tumour \\
\hline Subsacral
\end{tabular} \\
$\begin{array}{l}\text { Spinal dysraphism, } \\
\text { Familial dysautonomia }\end{array}$ & $\begin{array}{l}\text { Cauda equina injury, } \\
\text { Pelvic nerve injury }\end{array}$ & $\begin{array}{l}\text { Tumour, } \\
\text { Peripheral neuropathy } \\
\text { (e.g. diabetic) }\end{array}$ \\
\hline
\end{tabular}

Table 1: categorisation of neurological lesions according to time of onset, clinical course and CNS location, with example conditions.

*Conditions that can arise in more than one region of the CNS. 


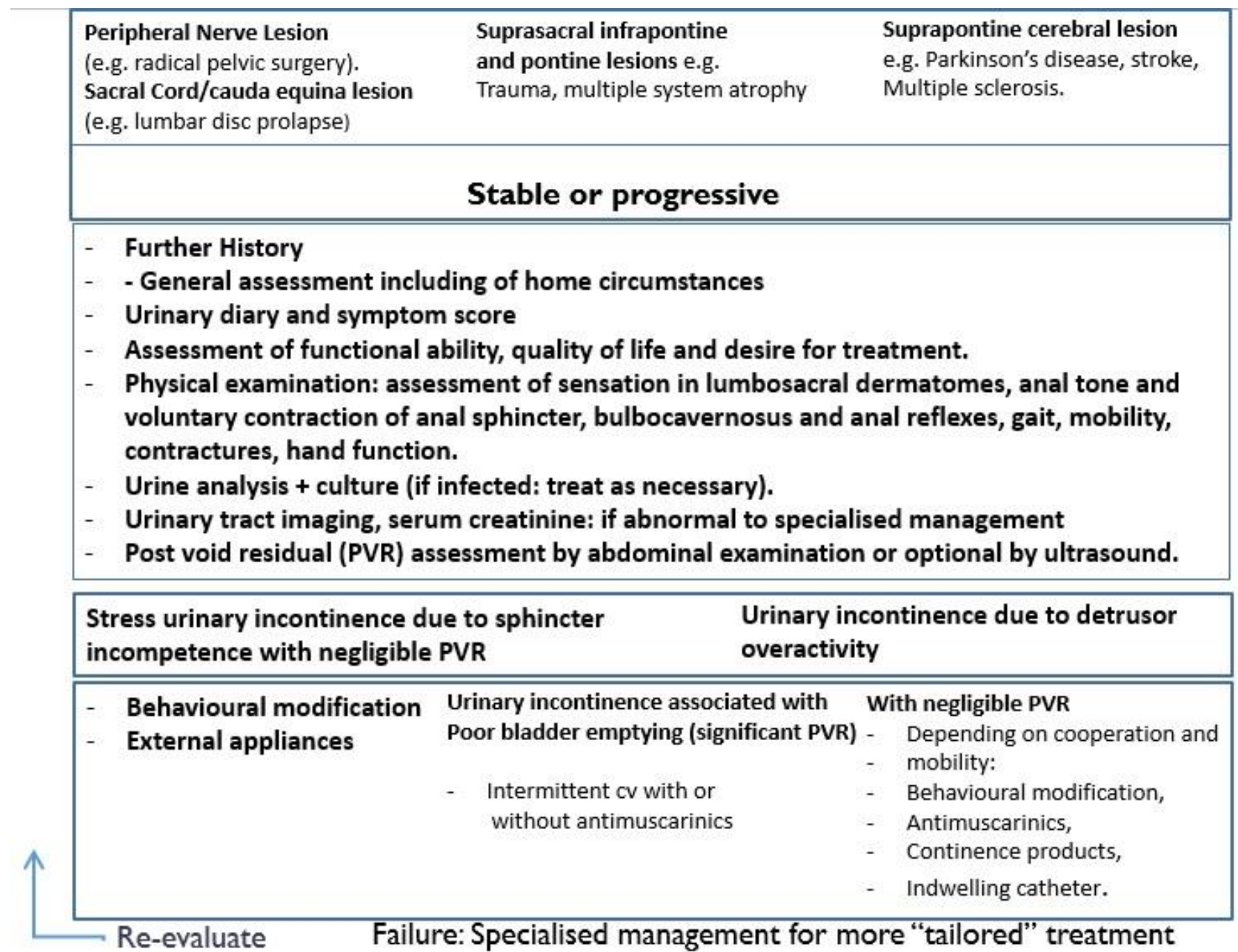

Figure 1; Initial assessment and therapy of neurogenic lower urinary tract dysfunction 


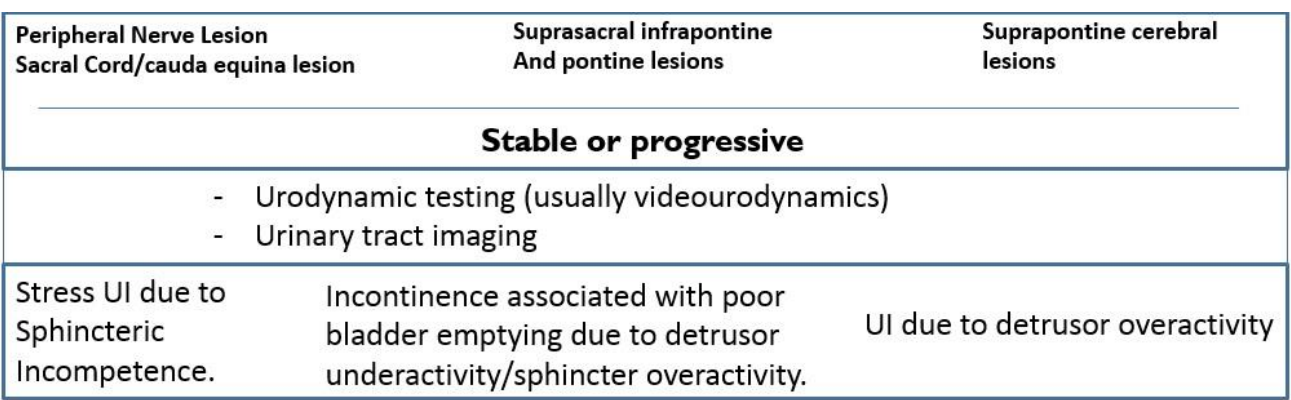

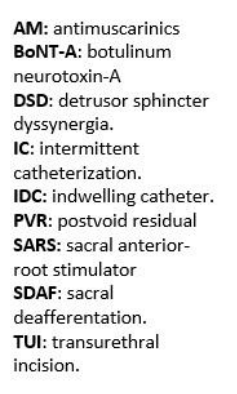

\begin{tabular}{|c|c|c|c|}
\hline & & With DSD & No DSD \\
\hline $\begin{array}{l}\text { Timed voiding } \\
\text { External appliance }\end{array}$ & $\begin{array}{ll}\text { - } & \text { IC } \\
\text { - } & \text { Alpha-1 blocker } \\
\text { - } & \text { Straining }\end{array}$ & $\begin{array}{ll}- & \text { IC + AM } \\
- & \text { IDC + AM } \\
- & \text { BoNT-A to detrusor } \\
& +I C\end{array}$ & $\begin{array}{ll}\text { - } & \text { Behavioural } \\
\text { - } & \text { IC + AM } \\
\text { - } & \text { Triggered voiding } \\
\text { - } & \text { Indwelling cath + } \\
& \text { AM } \\
\text { - } & \text { Botulinum Toxin to } \\
& \text { detrusor }\end{array}$ \\
\hline $\begin{array}{ll}\text { - } & \text { Artificial sphincter } \\
\text { - } & \text { Bladder neck sling } \\
\text { - } & \text { Autologous sling } \\
\text { - } & \text { Bulking agents } \\
\text { - } & \text { Bladder neck } \\
\text { closure. } \\
\text { - } & \text { Synthetic } \\
& \text { midurethral tapes. }\end{array}$ & $\begin{array}{ll}\text { - } & \text { Stents intraurethral. } \\
\text { - } & \text { TUI spincter. } \\
\text { - } & \text { Botulinum toxin to sphincter. }\end{array}$ & $\begin{array}{ll}- & \text { SDAF + IC } \\
\text { - } & \text { SDAF + SARS } \\
\text { - } & \text { Enterocystoplasty/Auto } \\
& \text { augmentation } \\
\text { - } & \text { Stents intraurethral. } \\
- & \text { TUl spincter. } \\
- & \text { Botulinum toxin to } \\
& \text { sphincter. }\end{array}$ & $\begin{array}{l}\text { Enterocystoplasty/ } \\
\text { Autoaugmentation }\end{array}$ \\
\hline
\end{tabular}

Stoma/diversion may be an option in selected cases

Figure 2; Specialised assessment and therapy of neurogenic lower urinary tract dysfunction 